\title{
Energy-Exchange Interactions between Colliding Vector Solitons
}

\author{
Charalambos Anastassiou, ${ }^{1}$ Mordechai Segev, ${ }^{1,2}$ Ken Steiglitz ${ }^{3}$ J. A. Giordmaine, ${ }^{1,4}$ Matthew Mitchell, ${ }^{1,5}$ \\ Ming-feng Shih, ${ }^{6}$ Song Lan, ${ }^{1}$ and John Martin ${ }^{7}$ \\ ${ }^{1}$ Electrical Engineering Department, Princeton University, Princeton, New Jersey 08544 \\ ${ }^{2}$ Physics Department, Technion-Israel Institute of Technology, Haifa 32000, Israel \\ ${ }^{3}$ Computer Science Department, Princeton University, Princeton, New Jersey 08544 \\ ${ }^{4}$ NEC Research Institute, Princeton, New Jersey 08540 \\ ${ }^{5}$ Lucent Technologies, Holmdel, New Jersey 07733 \\ ${ }^{6}$ Department of Physics, National Taiwan University, Taipei, Taiwan 10764 \\ ${ }^{7}$ Deltronic Crystal Industries, Dover, New Jersey 07801
}

(Received 21 April 1999)

\begin{abstract}
We demonstrate experimentally collisions between vector (Manakov-like) solitons that involve energy exchange at large collision angles, for which scalar solitons pass through one another practically unaffected.
\end{abstract}

PACS numbers: 42.65.Tg, 05.45.Yv

Vector solitons consist of two (or more) components that mutually self-trap in a nonlinear medium. They were first suggested by Manakov [1] for the Kerr nonlinearity, which is proportional to the intensity. The Manakov system leads to two coupled cubic nonlinear Schrödinger equations and is integrable and soluble analytically. Temporal Manakov-like solitons were proposed [2] and demonstrated in single mode optical fibers [3], and spatial ones were demonstrated in planar waveguides [4]. Vector solitons were also suggested [5] and observed [6] in a dark-bright form: when one of the components is a bright soliton and the other dark. Following the discovery of photorefractive spatial solitons, vector (Manakov-like) solitons were also suggested in photorefractives. In contrast to the Kerr nonlinearity, the photorefractive nonlinearity is saturable, but coincides with the Kerr nonlinearity in the limit of very low intensities [7]. One form of vector solitons found in photorefractives is of particular interest, because it applies to any noninstantaneous nonlinearity and allows more than two components: vector solitons based on mutual incoherence between the vector constituents [8]. Observations of such two-component vector solitons followed soon thereafter in three realizations: bright-bright, dark-dark, and dark-bright coupled pairs [9]. Finally, vector solitons can be realized as multimode [2,10] and multihump solitons which were recently demonstrated experimentally using the mutual-incoherence method [11].

Interactions between solitons are fascinating, since in many aspects solitons interact like particles: they pass through one another [12], undergo elastic collisions [13], and, in saturable nonlinearities [14], undergo fusion [15], fission and annihilation [16], and can even spiral about each other [17]. Soliton interactions depend on the number of soliton components. Thus far, interactions between vector solitons were studied theoretically only $[1,18,19]$, with the exception of one experiment demonstrating a bound state between two dark-bright solitons [20].
Here, we demonstrate experimentally interactions between vector solitons, highlighting features that are nonexistent for scalar solitons in the same regime: energy exchange at large collision angles, that is, in the regime where scalar solitons simply pass through each other [12].

Consider the coupled nonlinear wave equations for the slowly varying amplitudes of two EM fields, $A(x, z)$ and $B(x, z)$, in a $(1+1) \mathrm{D}$ system in which $x$ and $z$ are the transverse and longitudinal coordinates, respectively,

$$
\begin{aligned}
& \left(\frac{\partial}{\partial z}-\frac{i}{2 k} \frac{\partial^{2}}{\partial x^{2}}\right) A(x, z)=\frac{i k}{n_{0}} \Delta n(I) A(x, z), \\
& \left(\frac{\partial}{\partial z}-\frac{i}{2 k} \frac{\partial^{2}}{\partial x^{2}}\right) B(x, z)=\frac{i k}{n_{0}} \Delta n(I) B(x, z) .
\end{aligned}
$$

Here $\omega$ is the frequency of the carrier wave, $n=n_{0}+\Delta n$ the refractive index $\left(n_{0}\right.$ the unperturbed index and $\Delta n$ the nonlinear change in the index), $k=\omega n_{0} / c$ the wave number, and $I=|A|^{2}+|B|^{2}$ the total intensity. In its most familiar form, $\Delta n=n_{2}\left(|A|^{2}+|B|^{2}\right)$, with $n_{2}$ being a constant, Eqs. (1) represent Manakov solitons [1]. Other physical forms of $\Delta n$ do exist, of which we note $\Delta n=\Delta n_{0} /\left(1+|A|^{2}+|B|^{2}\right)$, a saturable nonlinearity that represents, for example, photorefractive screening solitons in [7], and the refractive index in a homogeneously broadened two-level system of atoms coupled to an EM field.

To understand collisions between two scalar Kerr solitons it is useful to draw on the induced-waveguide description of solitons [21]. A scalar Kerr soliton induces a $\operatorname{sech}^{2}(x)$ waveguide structure with waveguide parameter $V=\sqrt{2}$ at the wavelength of the soliton that has induced it, $\lambda$, and is a single mode waveguide at that wavelength. The $\operatorname{sech}^{2}(x)$ index profile is reflectionless for any plane wave (at $\lambda$ ) incident at any nonzero angle upon it. Thus, two interacting scalar Kerr solitons at a nonzero collision angle simply pass through one another, each conserving its energy and propagation angle (linear momentum) [12]. 
Here we discuss interactions between Manakov solitons $[18,19]$ which do give rise to an exchange of energy. We consider collisions between two vector solitons, in which one soliton (soliton 1) initially (at $z=0$ ) has two field components $\left(A_{1}\right.$ and $\left.B_{1}\right)$, and the other soliton (soliton 2) starts with one field component only $\left(A_{2}\right)$, i.e., $B_{2}=0$. The solitons are well separated at $z=0$, labeled as input, so that $A_{1}$ and $B_{1}$ form a single vector soliton, and $A_{2}$ and $B_{2}$ form the other soliton, which is initially a scalar (single component) soliton. The total intensity in both solitons is identical: $\left|A_{1}(x, z=0)\right|^{2}+\mid B_{1}(x, z=$ $0)\left.\right|^{2}=\left|A_{2}(x, z=0)\right|^{2}=2$, the collision angle is $0.5^{\circ}$ and $\left|A_{1}(x, z=0)\right|^{2}=\left|B_{1}(x, z=0)\right|^{2}$. The result can be calculated analytically $[18,19]$ or numerically as shown in Fig. 1. The total intensity, shown in Fig. 1(a), stays constant throughout propagation as in the case of scalar solitons.

However, unlike for scalar Kerr solitons, the field components that make up vector Kerr solitons do exchange energy upon collision, as shown in Fig. 1(b) which shows the $B$ field. They do it symmetrically, so the total intensity in each soliton is conserved, and the waveguides induced by the solitons remain identical. Such an energyexchange interaction is unique to vector solitons and cannot exist for scalar solitons. Right after the collision $B_{1}$ gives almost half of its energy to $B_{2}$ and energy is given from $A_{2}$ to $A_{1}$ to compensate for the energy lost by $B_{1}$.

There are two additional important features of this energy exchange. (1) It is not dependent on the initial phases of the input fields, as can be shown analytically [19] (the intuition for this follows below); (2) it occurs for any collision angle, but its efficiency decreases with increasing angles and with decreasing ratio $\left|A_{1}\right|^{2} /\left|B_{1}\right|^{2}$.

We can draw on a direct analogy between vector soliton collision and the four-wave mixing in nonlinear
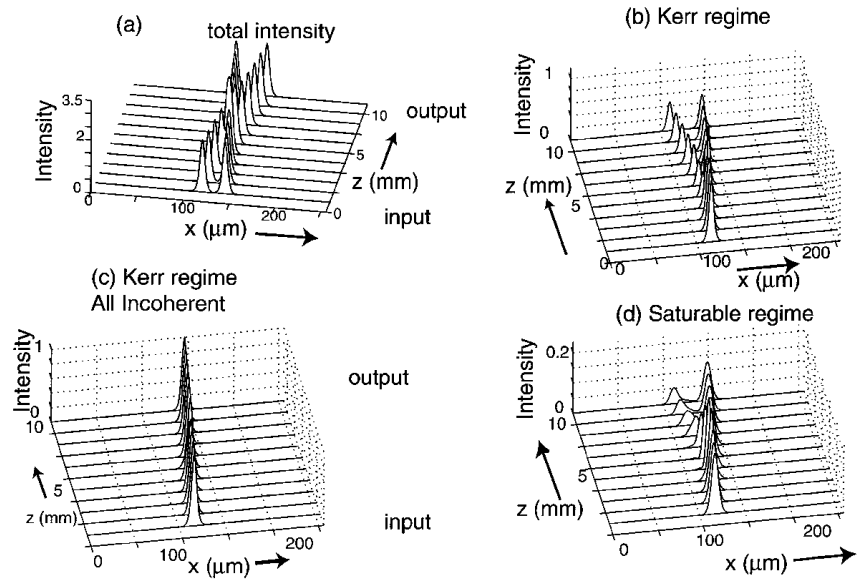

FIG. 1. Collisions of vector solitons at $0.55^{\circ}$ showing the total intensity (a) and the $B$ field for three different cases: for the Kerr regime and $A_{1}$ coherent with $A_{2}$ (b), for Kerr but all fields incoherent with each other-no grating (c), and for the saturable nonlinearity with $A_{1}$ and $A_{2}$ coherent (d). optics [22]: $A_{1}$ and $A_{2}$ (that are mutually coherent) form an interference grating, which is translated (via the nonlinearity) into a periodic index modulation with a grating vector $\mathbf{K}=\mathbf{k}_{1}-\mathbf{k}_{2}, \mathbf{k}_{1}$ and $\mathbf{k}_{2}$ corresponding to the propagation directions of $A_{1}$ and $A_{2}$. If the third input beam, $B_{1}$, travels in the $\mathbf{k}_{1}$ direction (as it does, because $A_{1}$ and $B_{1}$ form soliton 1), then it should Bragg diffract in the $\mathbf{k}_{2}$ direction and form a nonzero $B_{2}$. But $\mathbf{k}_{2}$ is the direction of $A_{2}$, so $B_{2}$ and $A_{2}$ together form soliton 2. This is the intuitive explanation for the energy-exchange interaction between vector solitons, and it takes place when the interference between $A_{1}$ and $A_{2}$ is translated into a periodic modulation of the index. The energyexchange interaction does not occur if $A_{1}$ and $A_{2}$ are made incoherent with one another, because the phase of the interference pattern fluctuates much faster than the nonlinear medium can respond to. Thus, no index grating forms and no energy exchange takes place. This is shown in Fig. 1(c): the solitons pass through each other, as if they were scalar solitons.

Since our experiments are in photorefractives, we set $\Delta n=\Delta n_{0} /\left(I+|A|^{2}+|B|^{2}\right)$. To avoid the strong transverse instability [which occurs for $(1+1) \mathrm{D}$ Kerr solitons in a bulk medium, and is suppressed in saturable nonlinearities if the total intensity is $>1$ [9]], we work at intensities of 2 . The collision angle is $0.55^{\circ}$, which is larger than the critical angle of the induced waveguide at this intensity, so scalar solitons simply go through each other [23]. For vector solitons in this medium energy exchange does take place [Fig. 1(d)], but it is less than the Manakov case even though we enhance the effect by increasing $\left|A_{1}\right|^{2} /\left|B_{1}\right|^{2}$ to 9 . If $A_{1}$ is made incoherent with $A_{2}$ the energy exchange is absent [as in the Manakov case shown in Fig. 1(c)].

Our experiments are carried out with screening solitons [7], utilizing the mutual-incoherence method to generate two-component vector solitons [9]. Our setup is shown in Fig. 2. We expand and collimate an $\mathrm{Ar}^{+}$laser beam, and then split it to ordinary, $o$, and extraordinary, $e$, polarized beams using a polarizing beam splitter. The $o$ and $e$ beams are polarized perpendicular and parallel, respectively, to the optical or $c$ axis of the crystal. The

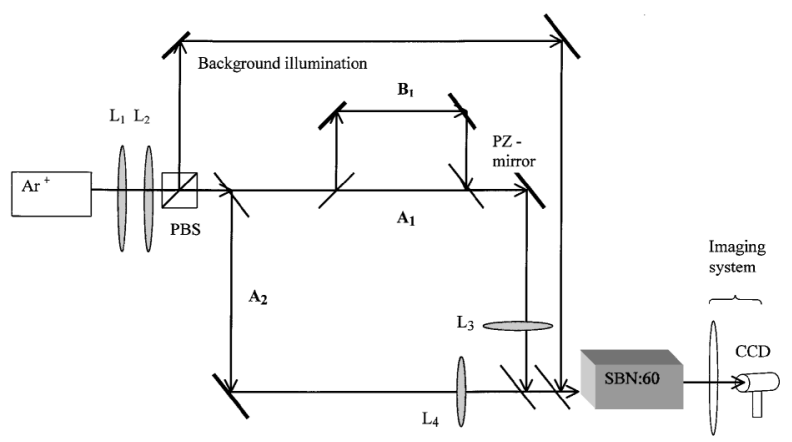

FIG. 2. Experimental setup. 
$o$ beam is used as a background illumination covering the crystal uniformly, as necessary for screening solitons $[9,11,23]$. The $e$ beam is then split into three, $A_{1}, A_{2}$, and $B_{1}$, which is made incoherent with $A_{1}$ and $A_{2}$ by having the optical length difference $(1 \mathrm{~m})$ exceed the coherence length of the laser $(10 \mathrm{~cm})$. Since the phase of $B_{1}$ is varying much faster than the response time of the crystal $\left(\tau_{d} \approx 1 \mathrm{sec}\right)$, no stationary interference pattern forms between $B_{1}$ and $A_{1}$ or $A_{2}$, since these beams are incoherent on the time frame of the crystal response [9]. $A_{1}$ and $B_{1}$ are then combined (using a beam splitter) and form soliton 1: they are focused with a cylindrical lens $L_{3}$ on the input face of a $1 \mathrm{~cm}$ long $\mathrm{SrBa}_{0.6} \mathrm{Nb}_{0.4} \mathrm{O}_{3}$ crystal. $A_{2}$ (which forms soliton 2) is also focused on the crystal using an identical lens, $L_{4}$. The input and output faces of the crystal are imaged on a CCD camera. The slow response of the crystal enables us to view each beam individually by blocking one beam (with a mechanical shutter) and sampling the other within a time interval $(\sim 1 \mathrm{msec})$ shorter than $\tau_{d}$. In this way [11] we distinguish between the individual field components of beams 1 and 2, even though they have the same wavelength and polarization. In order to compare the results of the energy-exchange interaction with the results of a collision in which all three input beams represent three independent fields [as those in Fig. 1(c)], we need a means to destroy the mutual coherence between $A_{1}$ and $A_{2}$. To do that, we insert a piezoelectric (PZ) mirror on the optical path of $A_{1}$. When the $\mathrm{PZ}$ mirror is driven by a square wave at a frequency of $\sim 2 \mathrm{kHz}$, the interference pattern formed by $A_{1}$ and $A_{2}$ varies faster than $\tau_{d}$ and thus does not contribute to the refractive index change. In other words, we expect energy exchange when the $\mathrm{PZ}$ mirror is "OFF," whereas when the PZ mirror is "ON" there is no index grating and we expect no energy exchange. Finally, a dc electric field is applied against the $c$ axis of the crystal for the formation of photorefractive screening solitons $[9,11,17,23]$.

In the first experiment shown in Fig. 3 we launch $15 \mu \mathrm{m}$ FWHM solitons colliding at an angle of $0.7^{\circ}$ (inside the crystal) with a total intensity ratio of 2 (normalized to the background intensity) for each soliton, $\left|A_{1}\right|^{2}=$ $3\left|B_{1}\right|^{2},\left|A_{2}\right|^{2}$ slightly higher than $\left|A_{1}\right|^{2}$, and $\left|B_{2}\right|^{2}=0$. The total intensity input is shown in (a), and the intensities of the individual field constituents are shown in (b) and (c). The normally diffracting output is shown in (d). When $700 \mathrm{~V}$ are applied between the electrodes separated by $0.5 \mathrm{~cm}$, the beams form solitons [(e)]. Note that, at the input (b), $A_{2}$ is on the left of $A_{1}$, whereas at the output (e), $A_{2}$ is on the right (the beams cross each other). (f) shows that $\sim 25 \%$ of field $B$ has transferred to soliton 2 (note the different scale for the $B$ output). At the same time, a small fraction of the energy of $A_{2}$ transfers to $A_{1}[(\mathrm{~g})]$. We emphasize that the coexistence of all three input beams $\left(A_{1}, A_{2}\right.$, and $B_{1}$ ) is required for this energy-exchange interaction: for

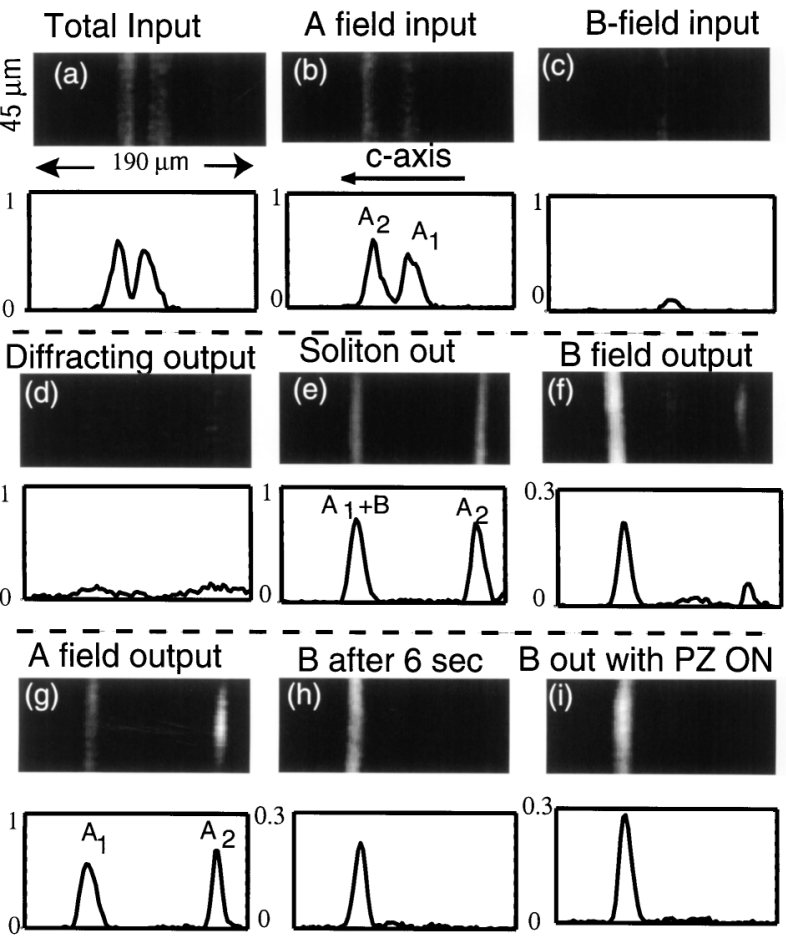

FIG. 3. Collisions at an angle of $0.7^{\circ}$ of $15 \mu \mathrm{m}$ FWHM solitons with a total intensity ratio of 2 . The pictures were taken with a CCD camera at the input and output faces of the crystal. (a) Total input. (b) $A$ field (consisting of $A_{1}$ and $A_{2}$ ). (c) $B$ field. (d) Diffracting output after $1 \mathrm{~cm}$ of propagation. (e) Output when the soliton forms. (f) $B$-field output when $A$ is blocked with a mechanical shutter showing coupling. (g) $A$-field output when $B$ is blocked. (h) The coupling disappears after $A$ remains blocked and the grating is washed away. (i) with the PZ mirror "ON" no coupling of energy occurs because all beams are incoherent.

example, if we establish the energy exchange and then block $A$ for a long time $\left(>\tau_{d}\right)$, this effect disappears, as shown in (h). In the spirit of Fig. 1(c), if the three input beams are fully incoherent with one another, there is no energy exchange because there is no refractive index grating (and the collision angle is larger than the critical angle of the waveguide induced by the solitons). The result in (i) shows the same experiment as in (f) but with the PZ mirror ON, confirming that, at these angles of collision, the energy-exchange interaction does not occur for three independent beams.

We then increase the collision angle to $0.9^{\circ}$. As expected from simulations, at large angles the energy switching should decrease. And indeed, the energy switching shown in Fig. 4(a) is now $\sim 10 \%$, as compared to $25 \%$ for the $0.7^{\circ}$ angle [Fig. 3(f)]. Finally, recalling the analogy with four-wave mixing, we realize that the modulation depth of the grating is proportional to the visibility of the interference, that is, to $A_{1} A_{2}^{*} / I, I$ being the total intensity. Therefore, keeping $I$ fixed, the visibility (and thus the energy switching efficiency) depends on the ratio $\left|A_{1}\right|^{2} /\left|B_{1}\right|^{2}$ at the input. We investigate this 


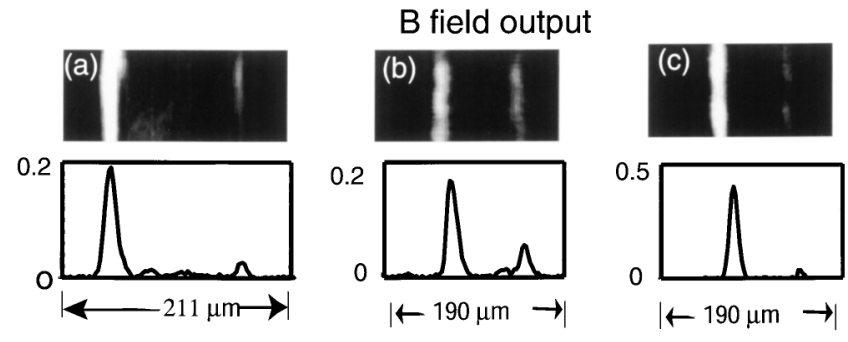

FIG. 4. $\quad B$ field right after collision. (a) Same as in Fig. 3(f) but with a collision angle of $0.9^{\circ}$ showing less coupling for $B$. (b),(c) Same as (a) with a collision angle of $0.55^{\circ}$, and different ratios $5\left|A_{1}\right|^{2}=\left|B_{1}\right|^{2}$ and $5\left|A_{1}\right|^{2}=\left|B_{1}\right|^{2}$, respectively, while the total intensity is kept constant. Comparing (b) to (c) we see more coupling when $A_{1}$ is stronger than $B_{1}$.

by comparing results with a collision angle of $0.55^{\circ}$ and two ratios: $\left|A_{1}\right|^{2}=5\left|B_{1}\right|^{2}$ and $5\left|A_{1}\right|^{2}=\left|B_{1}\right|^{2}$, shown in Figs. 4(b) and 4(c), respectively. Comparing Figs. 4(b) and 4(c) we notice that indeed the energy switching is much larger when $A_{1}$ is stronger than $B_{1}$, because the visibility is much larger.

In doing these experiments with photorefractive solitons, one must avoid effects from ordinary two-wave mixing that exists in photorefractives [22], and can lead to energy exchange in a preferential crystalline direction (towards the $c$ axis in our SBN crystal; the direction of the fanning). To avoid these effects, we arranged the energy exchange from $B_{1}$ to $B_{2}$ to be against the $c$ axis, i.e., against the direction of photorefractive two-wave mixing. So, all the effects we observe here truly result from soliton collisions and not from a specific photorefractive effect.

In conclusion, we have demonstrated experimentally energy-exchange interactions of vector solitons which are not possible for scalar solitons. This work suggests the possibility of generating phase conjugation of solitons, by having the field $B_{1}$ propagating counter to $A_{1}$. This leads to many other ideas, but we would like to end with just one: this interaction of vector solitons lays the experimental foundations for computation with solitons [19].

The work at Princeton was supported by the U.S. Army Research Office and by the NSF.

[1] S. V. Manakov, Sov. Phys. JETP 38, 248 (1974).

[2] D. N. Christodoulides and R. I. Joseph, Opt. Lett. 13, 53 (1988); M. V. Tratnik and J.E. Sipe, Phys. Rev. A 38, 2011 (1988); C. R. Menyuk, J. Opt. Soc. Am. B 5, 392 (1988).

[3] Y. Barad and Y. Silberberg, Phys. Rev. Lett. 78, 3290 (1997).

[4] J. U. Kang, G. I. Stegeman, J.S. Aitchison, and N. Akhmediev, Phys. Rev. Lett. 76, 3699 (1996).
[5] D. N. Christodoulides, Phys. Lett. A 132, 451 (1998); S. Trillo, S. Wabnitz, E. M. Wright, and G. I. Stegeman, Opt. Lett. 13, 871 (1988); V. V. Afanasjev, Y. S. Kivshar, V. V. Konotop, and V.N. Serkin, Opt. Lett. 14, 805 (1989).

[6] M. Shalaby and A. J. Barthelemy, IEEE J. Quantum Electron. 28, 2736 (1992).

[7] M. Segev, G.C. Valley, B. Crosignani, P. DiPorto, and A. Yariv, Phys. Rev. Lett. 73, 3211 (1994); D. N. Christodoulides and M. I. Carvalho, J. Opt. Soc. Am. B 12, 1628 (1995).

[8] D. N. Christodoulides, S. R. Singh, M. I. Carvalho, and M. Segev, Appl. Phys. Lett. 68, 1763 (1996).

[9] Z. Chen, M. Segev, T. Coskun, and D. N. Christodoulides, Opt. Lett. 21, 1436 (1996); 21, 1821 (1996); J. Opt. Soc. Am. B 14, 3066 (1997).

[10] M. Haelterman, A.P. Sheppard, and A. W. Snyder, Opt. Lett. 18, 1406 (1993); A. W. Snyder, S. J. Hewlett, and D. J. Mitchell, Phys. Rev. Lett. 72, 1012 (1994).

[11] M. Mitchell, M. Segev, and D. N. Christodoulides, Phys. Rev. Lett. 80, 4657 (1998).

[12] N. J. Zabusky and M. D. Kruskal, Phys. Rev. Lett. 15, 240 (1965).

[13] J. S. Aitchison, A. M. Weiner, Y. Silberberg, D. E. Leaird, M. K. Oliver, J. L. Jackel, and P. W. Smith, Opt. Lett. 16, 15 (1991); M. Shalaby, F. Reynaud, and A. Barthelemy, Opt. Lett. 17, 778 (1992).

[14] Fusion of solitons was predicted by S. Gatz and J. Herrmann, IEEE J. Quantum Electron. 28, 1732 (1992). Fission and annihilation of solitons were predicted by A. W. Snyder and A.P. Sheppard, Opt. Lett. 18, 482 (1993). Soliton spiraling was first suggested by D. J. Mitchell, A.W. Snyder, and L. Polodian, Opt. Commun. 85, 59 (1991).

[15] V. Tikhonenko, J. Christou, and B. Luther-Davies, Phys. Rev. Lett. 76, 2698 (1996); M. Shih and M. Segev, Opt. Lett. 21, 1538 (1996); Y. Baek, R. Schiek, G. I. Stegeman, and W. Sohler, Opt. Lett. 22, 1550 (1997).

[16] W. Krolikowski and S. A. Holmstrom, Opt. Lett. 22, 369 (1997); W. Krolikowski, B. Luther-Davies, C. Denz, and T. Tschudi, Opt. Lett. 23, 97 (1998).

[17] M. Shih, M. Segev, and G. Salamo, Phys. Rev. Lett. 78, 2551 (1997); A. Buryak, Y.S. Kivshar, M. Shih, and M. Segev, Phys. Rev. Lett. 82, 81 (1999).

[18] R. Radhakrishnan, M. Lakshmanan, and J. Hietarinta, Phys. Rev. E 56, 2213 (1997).

[19] M. H. Jakubowski, K. Steiglitz, and R. Squier, Phys. Rev. E 58, 6752 (1998).

[20] E. A. Ostrovskaya, Y.S. Kivshar, Z. Chen, and M. Segev, Opt. Lett. 24, 327 (1999).

[21] A. W. Snyder, D. J. Mitchell, and Y.S. Kivshar, Mod. Phys. Lett. B 9, 1479 (1995).

[22] A. Yariv, Optical Electronics (Wiley, New York, 1994).

[23] M. Shih, Z. Chen, M. Segev, T. Coskun, and D. N. Christodoulides, Appl. Phys. Lett. 69, 4151 (1996). 